\title{
ON RESIDUES OF POLYGENIC FUNCTIONS
}

\author{
BY \\ VINCENT C. POOR
}

1. Introduction. The class of complex functions considered here are defined in a domain $D$ of the complex plane. The single-valued function $f(z)$ $=u(x, y)+i v(x, y)$ is polygenic in the sense that the $z$ derivative

$$
\operatorname{limit}_{z \rightarrow z_{1}}\left(f(z)-f\left(z_{1}\right)\right) /\left(z-z_{1}\right)
$$

depends on the rectilinear direction of approach of $z$ to $z_{1}$. It, in fact, has a different value for each direction of approach.

In a previous paper in the Transactions [3] we considered residues at a point, of polygenic functions. Here our purpose is to extend these results to areas, generalizing the notion of classical residues of holomorphic functions. with the same object, of course, to characterize in some way polygenic functions. We shall develop the general theory and apply it to several special cases.

2. Character of problem and proposed solution. In the theory of monogenic functions the residue is defined by the equation

$$
R_{\sigma}=\frac{1}{2 \pi i} \int_{c} f(z) d z .
$$

If $f(z)$ has no poles inside the closed contour $c$ bounding the region $\sigma$, then $R_{\sigma}$ is zero. If in this region $F(z)$ has a pole so that $f(z)=F(z)(z-a)$, then the residue of $F(z)$

$$
R_{\sigma}=f(a) .
$$

The restriction here is that the contour $c$ bounding $\sigma$ includes no other singularities of $F(z)$.

If, on the other hand, $f(z)$ is a polygenic function, the contour integral is no longer independent of the path. Hence $R_{\sigma}$ given above depends on the contour $c$, and the definition loses its sense.

In the paper [3] referred to, we defined the residue of a polygenic function as the limit toward which the above expression, when the contour is regarded as a circle with radius $r$, center at $a$, tends when $r \rightarrow 0$. This definition generalizes the above definition for monogenic functions since for such functions it furnishes the same value of $R$.

It appears that there exists another way out of this difficulty which has an advantage over the "limit definition" for residues. We may note the fact

Presented to the Society, April 17, 1948: received by the editors March 17, 1952. 
that the failure in the application of the contour integral to polygenic functions is due to two distinct causes; one is that a polygenic function involves a certain continuously distributed density over the area and the other is the presence of discontinuities. The use of the residue is to characterize the discontinuity - we want therefore to eliminate the first cause. In the "limit definition" this purpose was achieved by contracting the contour and thus reducing the area to zero. In the alternative we are proposing, the same purpose is achieved by subtracting from the contour integral an area integral which accounts for the continuous distribution. The advantage is that the new definition conforms better to the general idea of residue - the value of the expression is now independent of the precise region over which we integrate; this is analogous to the classical residue. In the case considered the integral over the area will contain the Pompeiu [2] areal derivative as a factor in its integrand.

The following question now arises: Does the new definition give the same result as the "limit definition"? This paper is devoted to a study of the new definition and its extension and in particular to a discussion of the question just stated.

3. Basic theorems. Some fundamental theorems included here will be useful in the sequel.

THEOREM 1. If $f(z)$ possesses a differential at every point of $D$ so that $d f=f_{x} d x+f_{y} d y$, then for the circulation $\int_{c} f(z) d z$ around every rectifiable closed curve $c$ in $D$ bounding the area $\sigma$ to exist and be finite it is necessary and sufficient that the Pompeiu areal derivative $\partial f / \partial \alpha$ exist and be finite at every point of $D$.

The necessity. The two displacements $d z$ and $\delta z$ of the point $z$ define an elementary parallelogram, $\Delta \sigma_{j}$, say, whose boundary we designate by $c_{j}$. The flow around $c_{j}$ (area to the left) of $f(z)$ we compute starting with $f(z)$ at the point $z$. It follows then that

$$
\begin{aligned}
\int_{c_{j}} f(z) d z & =f(z) d z+\left(f(z)+d f+\eta_{1}\right) \delta z-f(z) \delta z-\left(f(z)+\delta f+\eta_{2}\right) d z \\
& =i\left[u_{x}-v_{y}+i\left(v_{x}+u_{y}\right)\right](d x \delta y-d y \delta x)+\eta_{1} \delta z-\eta_{2} d z \\
& =i\left[u_{x}-v_{y}+i\left(v_{x}+u_{y}\right)\right]\left(\Delta \sigma_{j}\right)^{\prime}+\eta_{1} \delta z-\eta_{2} d z,
\end{aligned}
$$

$\eta_{1}$ and $\eta_{2}$ being second order infinitesimal functions of $d x$ and $\delta y$ and $\left(\Delta \sigma_{j}\right)^{\prime}$ an infinitesimal area of the same order as $\Delta \sigma_{j}$. Hence

$$
\begin{aligned}
& \frac{1}{2 i \Delta \sigma_{j}} \int_{c_{j}} f(z) d z \\
& \quad=\frac{1}{2}\left[u_{x}-v_{y}+i\left(v_{x}+u_{y}\right)\right] \frac{\left(\Delta \sigma_{j}\right)^{\prime}}{\Delta \sigma_{j}}+\frac{1}{2 i \Delta \sigma_{j}}\left(\eta_{1} \delta z-\eta_{2} d z\right),
\end{aligned}
$$

and in the limit as $\Delta \sigma_{j} \rightarrow 0$ as an area the left member of (3.2) becomes the 
areal derivative at $z$ while the terms in $\eta_{1}$ and $\eta_{2}$ vanish so that

$$
\partial f / \partial \alpha=\left[u_{x}-v_{y}+i\left(v_{x}+u_{y}\right)\right] / 2 .
$$

Thus the areal derivative exists and is finite at every point of $D$.

The sufficiency. We here choose a rectifiable closed curve $c$ in $D$ bounding the area $\sigma$. We then divide $\sigma$ up into a system of $n$ mesh circuits by two families of intersecting parallel lines. For the $j$ th mesh we have by definition

$$
\frac{\partial f}{\partial \alpha}=\operatorname{limit}_{\Delta \sigma_{j} \rightarrow 0} \frac{1}{2 i \Delta \sigma_{j}} \int_{o_{j}} f(z) d z
$$

from this it follows that

$$
\frac{1}{2 \pi i} \int_{c_{j}} f(z) d z=\frac{1}{\pi} \frac{\partial f}{\partial \alpha} \Delta \sigma_{j}+\eta_{j} \Delta \sigma_{j}
$$

where $\eta_{j}$ is an infinitesimal function of $\Delta \sigma_{j}$. We now sum the terms of the last equation $(j=1,2, \cdots, n)$ and observe that $\sum_{j=1}^{n} \eta_{j} \Delta \sigma_{j}$ goes to zero as $n$ increases without limit by the Duhamel theorem. By the fundamental law of the integral calculus the remaining terms lead to the equation

$$
\frac{1}{2 \pi i} \int_{c} f(z) d z=\frac{1}{\pi} \int_{\sigma} \frac{\partial f}{\partial \alpha} d \sigma .
$$

However, in computing the left member of (3.3) we resort to the use of the triangles formed by drawing a diagonal of each parallelogram, together with those triangles at the boundary one side of each of which is the join of two adjacent points of intersection of the families of lines with the curve $c$. These joins form an $n$-gon.

Now, the circulation around a parallelogram is equal to the sum of the circulations around its two triangles since the resultant flow along the diagonal is zero. The resultant flow along a common side of two adjacent triangles is zero. Thus the resultant circulation is the flow around the $n$-gon. In the limit then as $n \rightarrow \infty$ this becomes the circulation integral taken around $c$, since for a rectifiable curve the ratio of the cord to the subtended arc is unity. If in the right member the summation is by triangles the limit will evidently be the integral over the area. The areas between the cords and their subtended arcs are second order infinitesimals which also vanish in the limit.

This proves the theorem since the right member of (3.3) exists and is finite.

In passing we include the

COROLlary. If $f(z)$ is continuous, the necessary and sufficient condition that $f(z)$ be analytic in $D$ is that the areal derivative $\partial f / \partial \alpha=0$ at every point of $D$.

If $f(z)$ is analytic in $D$, then evidently the areal derivative vanishes. The 
converse of this is the Morera theorem since $f(z)$ is continuous.

Theorem 2. If $f(z)$ possesses a differential of the form $d f=f_{x} d x+f_{y} d y$, then for the circulation $\int_{c} f(z) d \bar{z}$ to exist and to be finite for every rectifiable closed curve $c$ in $D$ bounding $\sigma$, it is necessary and sufficient that the Kasner mean derivative [1] $\partial f / \partial \beta$ exist and be finite at every point of $D$.

The analogue to the corollary above may be stated and proved. The vanishing of the mean derivative makes $f(z)$ analytic in $\bar{z}$. The proof of Theorem 2 will be omitted since the proof may follow the arguments on Theorem 1.

The mean derivative is defined by the equation

$$
\frac{\partial f}{\partial \beta}=\operatorname{limit}_{\Delta \sigma_{j} \rightarrow 0} \frac{-1}{2 i \Delta \sigma_{j}} \int_{c_{j}} f(z) d \bar{z}
$$

where $\bar{z}$ as usual is the conjugate of $z$. However, Theorem 2 leads at once to the theorem

$$
-\frac{1}{2 \pi i} \int_{c} f(z) d \bar{z}=\frac{1}{\pi} \int_{0} \frac{\partial f}{\partial \beta} d \sigma .
$$

4. Residues of polygenic functions. Following the suggestions in $\$ 2$ we define the residue of $f(z)$ over an area $\sigma$ in $D$ by the equation:

$$
R^{\prime}=\frac{1}{2 \pi i} \int_{c} f(z) d z-\frac{1}{\pi} \int_{\sigma} \frac{\partial f}{\partial \alpha} d \sigma\left(^{(1)}\right.
$$

when these integrals have a meaning.

It is assumed throughout that the areal (mean) derivative of the polygenic function $f(z)$ in general exists and is finite in $D$; this statement is omitted when it is clear from the context.

THEOREM 3. If $f(z)$ is continuous and if the areal derivative exists and is finite in $D$, then the residue $R^{\prime}$ over $\sigma$ bounded by $c$ in $D$ is zero.

This theorem follows directly from (3.3). If $f(z)$ is analytic in $D, R^{\prime}$ reduces to the classical residue since in this case $\partial f / \partial \alpha=0$.

In the sequel we shall consider a function $f(z)$ to be regular [4] in the sense that it possesses a restricted Hamilton differential so that $f(z)$ may be expressed in the form

$$
f(z)=f(a)+\frac{\partial f}{\partial \beta}(z-a)+\frac{\partial f}{\partial \alpha}(\bar{z}-\bar{a})+\eta
$$

where $\eta$ is a second order infinitesimal function of $z-a$, or it may be considered "der Rest der Reihe" of a Taylor series [5].

(1) This result was communicated to me by Professor I. Halperin in a letter now misplaced. 
Theorem 4. If $f(z)$ is regular at every point of $D$ and if $z=a$ is a single simple isolated pole of $F(z)$ in $\sigma$ bounded by $c$ a rectifiable closed curve in $D$ so that $f(z)=(z-a) F(z)$, then the residue $R^{\prime}$ of $F(z)$ over $\sigma$ is

$$
R_{\sigma}(F)=\frac{1}{2 \pi i} \int_{c} \frac{f(z) d z}{z-a}-\frac{1}{\pi} \int_{\sigma} \frac{\partial f / \partial \alpha}{z-a} d \sigma=f(a) .
$$

This is the Pompeiu theorem [2]. However it is easily proved by surrounding the point $a$ by a circle center at $a$ and applying (3.3) to the annular area and contracting the circle to a point. It is understood that the circle is joined to $c$ by a line making the annular area simply connected. The integrals along the connection cancel so need not appear in the analysis. Again if $f(z)$ is analytic in $D$ the integral over $\sigma$ drops out leaving the classical residue.

This, of course, is not the whole story for a second type of residue arises for polygenic functions. This type of residue is associated with the mean derivative. We define it by the equation

$$
\bar{R}^{\prime}=-\frac{1}{2 \pi i} \int_{c} f(z) d \bar{z}-\frac{1}{\pi} \int_{\sigma} \frac{\partial f}{\partial \beta} d \sigma
$$

when these integrals have a meaning.

THEOREM 5. If $f(z)$ is continuous and if the mean derivative exists and is finite in $D$, then the residue $\bar{R}^{\prime}$ of $f(z)$ over $\sigma$ bounded by $c$ in $D$ is zero.

This is evidently a consequence of (3.4).

THEOREM 6. If $f(z)$ is regular at every point of $D$ and if $z=a$ is a single simple isolated pole of $F(z)$ in $\sigma$ bounded by a rectifiable closed curve $c$ in $D$ so that $f(z)=(z-a) F(z)$, then the residue $\bar{R}^{\prime}$ of $F(z)$ over $\sigma$ is zero.

In the proof of this theorem we surround the point $a$ by a circle, radius $r$ with center at $a$, and write out $\bar{R}^{\prime}$ for the annular area $\sigma^{\prime}$ between $c$ and $o$. We thus have

$$
\bar{R}_{\sigma^{\prime}}^{\prime}(F)=-\frac{1}{2 \pi i} \int_{c} \frac{f(z) d \bar{z}}{z-a}+\frac{1}{2 \pi i} \int_{0} \frac{f(z) d \bar{z}}{z-a}-\frac{1}{\pi} \int_{\sigma^{\prime}} \frac{\partial f}{\partial \beta} \frac{f(z)}{z-a} d \sigma=0
$$

since the annular area contains no discontinuity. The integral around the circle

$$
\begin{aligned}
\int_{0} \frac{f(z) d \bar{z}}{z-a}= & f(a) \int_{0} \frac{d \bar{z}}{z-a}+\frac{\partial f}{\partial \beta} \int_{0} \frac{z-a}{z-a} d \bar{z} \\
& +\frac{\partial f}{\partial \alpha} \int_{0} \frac{(\bar{z}-\bar{a}) d \bar{z}}{z-a}+\int_{0} \frac{\eta d \bar{z}}{z-a} .
\end{aligned}
$$

The first three integrals on the right of (4.6) are identically zero while the 
last goes to zero with $r$ since $\eta$ is a second order infinitesimal function of $z-a=r e^{i \theta}$. Hence as $\sigma^{\prime} \rightarrow \sigma, \bar{R}^{\prime} \rightarrow 0$.

We now define the total residue by the equation

$$
R=R^{\prime}+\bar{R}^{\prime} .
$$

Thus if $f(z)$ is continuous and the integrand has no singularities,

$$
R=0 \text {, }
$$

while if the integrand carries also the simple pole $z=a$ only, we see that

$$
R=R^{\prime}+\bar{R}^{\prime}=f(a) \text {. }
$$

5. Many isolated poles and poles of higher order. Suppose that $z=a_{1}$ and $z=a_{2}$ are single simple isolated poles in $\sigma$ so that $f(z)=F(z)\left(z-a_{1}\right)\left(z-a_{2}\right)$, where $f(z)$ is regular, as defined by (4.2). Then by definition

$$
R^{\prime}=\frac{1}{2 \pi i} \int_{c} \frac{f(z) d z}{\left(z-a_{1}\right)\left(z-a_{2}\right)}-\frac{1}{\pi} \int_{\sigma} \frac{(\partial f / \partial \alpha) d \sigma}{\left(z-a_{1}\right)\left(z-a_{2}\right)} .
$$

To show that these integrals exist and are finite over $\sigma$ we surround $a_{1}$ and $a_{2}$ by nonintersecting circles $o_{1}$ and $o_{2}$ with centers at $a_{1}$ and $a_{2}$ respectively. We join each of the circles to $c$ by straight lines, say, making the area between $c$ and the circles a simply-connected region, $\sigma^{\prime}$. The integrals along the joins cancel each other as before and do not appear. We then have for this area $\sigma^{\prime}$

$$
\begin{aligned}
R_{\sigma^{\prime}}^{\prime}= & \frac{1}{2 \pi i} \int_{c} \frac{f(z) d z}{\left(z-a_{1}\right)\left(z-a_{2}\right)}-\frac{1}{2 \pi i} \int_{o_{1}} \frac{f(z) d z}{\left(z-a_{1}\right)\left(z-a_{2}\right)} \\
& -\frac{1}{2 \pi i} \int_{o_{2}} \frac{f(z) d z}{\left(z-a_{1}\right)\left(z-a_{2}\right)}-\frac{1}{\pi} \int_{\sigma^{\prime}} \frac{(\partial f / \partial \alpha) d \sigma}{\left(z-a_{1}\right)\left(z-a_{2}\right)}=0 .
\end{aligned}
$$

If the function $f(z)$ is regular and treated as in Theorem 6 , it will be simple to show, and it was implied in Theorem 4, that the general result holds that

$$
\operatorname{limit}_{r \rightarrow 0} \frac{1}{2 \pi i} \int_{0} \frac{f(z) d z}{z-a}=f(a)
$$

$o$ being a circle with radius $r$ and center $z=a$.

For the circle around $o_{1}, f(z)\left(z-a_{2}\right)$ is the regular function in the integrand so that the second term on the right in (5.2) becomes $-f\left(a_{1}\right) /\left(a_{1}-a_{2}\right)$ and similarly the third integral becomes $-f\left(a_{2}\right) /\left(a_{2}-a_{1}\right)$ in the limits.

Hence the residue over $\sigma$

$$
R_{\sigma}^{\prime}=\frac{f\left(a_{1}\right)-f\left(a_{2}\right)}{a_{1}-a_{2}}
$$


since each circle has been contracted to a point, its center.

The result here generalizes at once to $n$ isolated simple poles $a_{j}$ $(j=1, \cdots, n)$. Evidently

$$
R^{\prime}=\sum_{j=1}^{n} \frac{f\left(a_{j}\right)}{\prod_{k=1, j \neq k}^{n}\left(a_{j}-a_{k}\right)} .
$$

It will also be easy to show that

$$
\bar{R}^{\prime}=0 .
$$

The analysis will be omitted.

Poles of higher order may be treated in some similar manner, each case on its own merits.

6. Singularities of a certain linear function. There was another type of pole considered in the Transactions paper. The problem there was to determine the residues at the origin of the function $(a z+b \bar{z})^{-1}$. We shall broaden this problem by introducing the factor $f(z)$, a regular polygenic function defined by (4.2). The problem here then is to determine the residues of $f(z)(a z+b \bar{z})^{-1}$ over an area $\sigma$ bounded by a rectifiable closed curve $c$ in $D$, with the origin a point of $\sigma$.

We surround the origin by a circle $o$, radius $r$ with center at the origin, and since the poles of the integrand are at the origin we have for the annular area $\sigma^{\prime}$ between $o$ and $c$

$$
R_{\sigma^{\prime}}^{\prime}=\frac{1}{2 \pi i} \int_{0} \frac{f(z) d z}{a z+b \bar{z}}-\frac{1}{2 \pi i} \int_{0} \frac{f(z) d z}{a z+b \bar{z}}-\frac{1}{\pi} \int_{\sigma^{\prime}} \frac{\partial}{\partial \alpha} \frac{f(z)}{a z+b \bar{z}} d \sigma=0
$$

with the similar defining equation for $\bar{R}_{\sigma}^{\prime}$. In evaluating the integral around the circle $o$ it is to be understood that the poles of the integrand, when they fall outside $o$, lie in the annular area.

When $f(o)$ replaces $f(z)$ in the integrand of the integral around the circle $o$ aside from the constant factor $f(o)$, we obtain the integral treated in the Transactions paper [3]. But the situation is different here; in the former paper poles outside the circle $o$ gave a zero contribution to the residues. In case I: $|b|>|a|$ the poles of the integrand fall in the annular area. In this case, since

$$
\frac{z}{a z^{2}+b r^{2}}=\frac{1}{2 a}\left(\frac{1}{z-i(b / a)^{1 / 2} r}+\frac{1}{z+i(b / a)^{1 / 2} r}\right)
$$

we must revise (6.1); we draw two nonintersecting circles $o_{1}$ and $o_{2}$ with centers at the poles; we connect the circles to $c$ by straight lines, say, to preserve the simply connected region. We subtract the integrals around these circles each multiplied by $1 / 2 \pi i$ from the left member of (6.1). It is evident from 
this that the residue

$$
R_{\sigma}^{\prime}=f(o) / a .
$$

The integral around $o$ is found and when each circle involved is collapsed to a point, $\sigma^{\prime}$ becomes $\sigma$. Here also $\bar{R}_{\sigma}^{\prime}=f(o) / b$ which is found by first writing the integrand in the form $(\bar{z} / b) /\left(\bar{z}^{2}+(a / b) r^{2}\right)$ which in turn has its two poles inside the circle. Had the poles in (6.2) been inside the circle, Case II : $|b|<|a|$ the residue $R_{\sigma}^{\prime}$ would have been the same. In Case III: $|b|$ $=|a|$, the poles were distributed along a straight line through the origin. Here the residues will differ from those in the former paper by the factor $f(0)$.

When $f(z)$ is replaced by $(\partial f / \partial \beta) z$, the integrand, omitting the constant factors, is

$$
\frac{z^{2}}{z^{2}+(b / a) r^{2}}=1+\frac{b r^{2}}{a\left(z^{2}+(b / a) r^{2}\right)}
$$

When $(\partial f / \partial \alpha) \bar{z}$ replaces $f(z)$, the integrand will be $r^{2} /\left(z^{2}+(b / a) r^{2}\right)$ omitting some constant factors again. When $|b| \neq|a| \neq 1$ we need consider only the function

$$
\frac{1}{z^{2}+(b / a) r^{2}}=\frac{a^{1 / 2}}{i b^{1 / 2} r}\left(\frac{1}{z-i(b / a)^{1 / 2} r}-\frac{1}{z+i(b / a)^{1 / 2} r}\right) .
$$

We treat the two poles here as formerly. The contribution to the residues will evidently be zero.

When $|b|=|a|$, Case III, we have to consider only the integral

$$
\int \frac{r^{2} d z}{\bar{z}^{2}+e^{i \phi^{2}}}
$$

The integrand will have poles distributed along the straight line with slope angle $(\phi-\pi) / 2$. But aside from the fact that the integral around $o$ is zero, there is an extra $r$ in the numerator which goes to zero also. Thus the second and third terms in $f$ contribute zero to the residue.

When $f(z)$ is replaced by $\eta$ in the integrand in the integral around $o$ in (6.1) and $\eta$ in turn by a homogeneous quadratic function, the integral reduces to the original integrals each multiplied by $r^{2}$ or $r$ with an exponent greater than two, and to some other integrals which are zero or carry the factor $r^{2}$. Higher degree terms than the second in $\eta$ act even more favorably. Thus $\eta$ contributes zero to the residues.

We determine $\bar{R}^{\prime}$ in the same way as indicated using $\bar{z}$ as the variable. The final results tabulate as follows:

$$
\text { Case I: } \quad|b|>|a| ; \quad R_{\sigma}^{\prime}=\frac{f(o)}{a}, \quad \bar{R}_{\sigma}^{\prime}=\frac{f(o)}{b} ; \quad R=f(o) \frac{a+b}{a b} ;
$$


Case II: $\quad|b|<|a| ; \quad R_{\sigma}^{\prime}=\frac{f(o)}{a} ; \quad \bar{R}_{\sigma}^{\prime}=\frac{f(o)}{b} ; \quad R=f(o) \frac{a+b}{a b} ;$ Case III: $|\dot{b}|=|a| ; \quad R_{\sigma}^{\prime}=\frac{f(o)}{2 a} ; \quad \bar{R}_{\sigma}{ }^{\prime}=\frac{f(o)}{2 b} ; \quad R=f(o) \frac{a+b}{2 a b}$.

7. Singularities of certain quadratic functions. We shall be interested here in the residues of the function $f(z)\left(a z^{2}+b_{z}^{\prime} z \bar{z}+c^{\prime} \bar{z}^{2}\right)^{-1}$ over an area $\sigma$ bounded by a rectifiable closed curve $c$ in $D$. The function $f(z)$ is defined by (4.2), while the origin is a point of $\sigma$. Without affecting the generality of the problem we take the constant $a$ equal to unity so that

$$
\begin{aligned}
\left(a z^{2}+b^{\prime} z \bar{z}+c^{\prime} \bar{z}^{2}\right)^{-1} & =z^{2}\left(z^{4}+b^{\prime} r^{2} z^{2}+c^{\prime} r^{4}\right)^{-1} \\
& =z^{2}\left(z^{2}+b_{1} r^{2}\right)^{-1}\left(z^{2}+b_{2} r^{2}\right)^{-1}
\end{aligned}
$$

when $r$ is constant.

If a circle $o$, radius $r$, is drawn with the origin as center the residue over the annular area $\sigma^{\prime}$ between $o$ and $c$,

$$
\begin{aligned}
R_{\sigma^{\prime}}^{\prime}= & \frac{1}{2 \pi i} \int_{c} \frac{f(z) d z}{z^{2}+b^{\prime} z \bar{z}+c^{\prime} \bar{z}^{2}}-\frac{1}{2 \pi i} \int_{0} \frac{f(z) z^{2} d z}{\left(z^{2}+b_{1} r^{2}\right)\left(z^{2}+b_{2} r^{2}\right)} \\
& -\frac{1}{\pi} \int_{\sigma^{\prime}} \frac{\partial}{\partial \alpha} \frac{f(z)}{z^{2}+b^{\prime} z \bar{z}+c^{\prime} \bar{z}^{2}} d \sigma=0,
\end{aligned}
$$

with the similar defining equation for $\bar{R}_{\sigma^{\prime}}^{\prime}$.

There are essentially nine different cases to consider and when $f(z)$ is replaced by the linear terms in (4.2) there will be three times as many, disregarding the number arising when $\bar{R}_{\sigma}^{\prime}$ is to be determined.

We, of course, concern ourselves with the integral around $o$ and its alter egos. If some of the poles are distinct and lie in the annular area, (7.1) has to be revised. We treat it and the integrals around the other circles needed just as in $\S 6$, and finally contract each circle to a point.

When $f(z)$ is replaced by $f(o)$, aside from the constant factors, the integrand of the integral around $o$

$$
\frac{z^{2}}{\left(z^{2}+b_{1} r^{2}\right)\left(z^{2}+b_{2} r^{2}\right)}=\frac{b_{1}}{b_{1}-b_{2}} \frac{1}{z^{2}+b_{1} r^{2}}+\frac{b_{2}}{b_{2}-b_{1}} \frac{1}{z^{2}+b_{2} r^{2}}\left(b_{1} \neq b_{2}\right)
$$

and when $b_{1}=b_{2}=b$ we have

$$
\frac{z^{2}}{\left(z^{2}+b r^{2}\right)^{2}}=\frac{1}{z^{2}+b r^{2}}-\frac{b r^{2}}{\left(z^{2}+b r^{2}\right)^{2}} .
$$

The residues here will be zero in all cases. Each integral excepting the last is essentially the function obtained in (6.3). It is easy to show that the last function when properly integrated will vanish also in all cases. When the 
poles are distributed along a straight line chief values must be taken, as in $\S 6$. A complete classification of cases will be given subsequently.

When $f(z)$ is replaced by $(\partial f / \partial \beta) z$ in (7.1) the integrand of the integral around $o$, omitting the constant factor, is

$$
\frac{z^{3}}{\left(z^{2}+b_{1} r^{2}\right)\left(z^{2}+b_{2} r^{2}\right)}=\frac{b_{1}}{b_{1}-b_{2}} \frac{z}{z^{2}+b_{1} r^{2}}+\frac{b_{2}}{b_{2}-b_{1}} \frac{z}{z^{2}+b_{2} r^{2}}, \quad b_{1} \neq b_{2},
$$

while

$$
\frac{z^{3}}{\left(z^{2}+b r^{2}\right)^{2}}=\frac{z}{z^{2}+b r^{2}}-\frac{b r^{2} z}{\left(z^{2}+b r^{2}\right)^{2}}, \quad b_{1}=b_{2}=b .
$$

These functions arose in $\S 6$ except the last in which case it is not reducible when the poles are linearly distributed.

We consider briefly one case: $b_{1}=e^{i \phi} ;\left|b_{2}\right|>1$. Since $b_{1} \neq b_{2}$ we use (7.4). The integrand of the first integral will have poles distributed along the straight line with slope angle $(\phi-\pi) / 2$ as in $\S 6$. The integral around $o$,

$$
\begin{aligned}
\int_{0} \frac{z d z}{z^{2}+e^{i \phi} \boldsymbol{r}^{2}} & =\int_{0}^{2 \pi} \frac{i e^{i \theta} d \theta}{e^{i \theta}+e^{i(\phi-\theta)}} \\
& =\frac{1}{2} \int_{0}^{2 \pi} i d \theta-\int_{0}^{2 \pi} \tan \left(\frac{\phi}{2}-\theta\right) d \theta=\pi i .
\end{aligned}
$$

In evaluating the second integral from $0 \rightarrow 2 \pi$ we take chief values avoiding the line of poles by integrating from $(\phi-\pi) / 2+\epsilon$ to $(\phi+\pi) / 2-\epsilon$ and from $(\phi+\pi) / 2+\epsilon$ to $(\phi-\pi) / 2-\epsilon$. When the terms are properly paired off, they will be seen to cancel in the limit as $\epsilon \rightarrow 0$.

The second function on the right in (7.4) has two poles in the annular area, which are to be treated exactly as in $\S 6$. The sum of the two integrals arising, omitting the constant factors, is $2 \pi i$. In this case the resultant residue

$$
R_{\sigma}^{\prime}=\frac{b_{1}-2 b_{2}}{2\left(b_{1}-b_{2}\right)} \frac{\partial f}{\partial \beta} \text {. }
$$

When $f(z)$ is replaced by $(\partial f / \partial \alpha) \bar{z}$ in the integrand of the integral around $o$ we obtain the function

$$
\frac{r^{2} z}{\left(z^{2}+b_{1} r^{2}\right)\left(z^{2}+b_{2} r^{2}\right)}=\frac{1}{b_{1}-b_{2}}\left(\frac{z}{z^{2}+b_{2} r^{2}}-\frac{z}{z^{2}+b_{1} r^{2}}\right), \quad b_{1} \neq b_{2},
$$

while for $b_{1}=b_{2}=b$ we get $r^{2} z /\left(z^{2}+b r^{2}\right)^{2}$, which is not reducible when $|b|=1$. In all cases $R_{\sigma}^{\prime}=0$ excepting in the two cases, II and VII below, where

$$
R_{\sigma}^{\prime}=\frac{\partial f}{\partial \alpha} / 2\left(b_{1} b_{2}\right)
$$


When $f(z)$ is replaced by $\eta$ in the integral around $o$ in (7.1) and it in turn by a homogeneous quadratic function, we shall have a situation analogous to that in $\S 6$. The statements there are also applicable here. The finite integrals already studied will recur each multiplied by $r$ with an exponent sufficiently large. The other integrals arising are zero or they go to zero with $r$. The contribution to the residues $R_{\sigma}^{\prime}$ furnished by $\eta$ is zero.

In tabulating the final results we have attempted to order the sets, working from inside the circle $o$ to the annular area, $b_{1}$ playing the leading role. Thus

CASES I, IV, IX: $\left|b_{1}\right| \lessgtr 1, \quad\left|b_{2}\right| \lessgtr 1, \quad b_{1} \neq b_{2} ; \quad R_{\sigma}^{\prime}=\frac{\partial f}{\partial \beta}$,

CASES II, VII: $\quad b_{1}=e^{i \phi},\left|b_{2}\right| \lessgtr 1 ; \quad R_{\sigma}^{\prime}=\frac{1}{2\left(b_{1}-b_{2}\right)}\left(\left(b_{1}-2 b_{2}\right) \frac{\partial f}{\partial \beta}+\frac{\partial f}{\partial \alpha}\right)$

CASES III, VIII: $\quad b_{1}=b_{2}=b, \quad|b| \lessgtr 1 ; \quad R_{\sigma}^{\prime}=\frac{\partial f}{\partial \beta}$,

CASE V: $\quad b_{1}=b_{2}=b=e^{i \phi} ; \quad R_{\sigma}^{\prime}=\frac{1}{2} \frac{\partial f}{\partial \beta}$,

CASE VI: $\quad b_{1}=e^{i \phi}, \quad b_{2}=e^{i \psi} ; \quad R_{\sigma}^{\prime}=\frac{1}{2} \frac{\partial f}{\partial \beta}$.

The similar results obtained for $\bar{R}_{\sigma}^{\prime}$ defined by (4.4) will be entirely omitted.

A second quadratic function is $(z \bar{z}-1)^{-1}$ with poles distributed on the circle radius one. We simplify the argument by drawing three circles with centers at the origin $o_{1}$ with radius $1-\epsilon, o_{2}$ with radius $1+\epsilon$, and $o_{3}$ with radius $r_{3}$ $>1+\epsilon$.

For the circular area $\sigma_{1}$ bounded by $o_{1}$ we have

$$
\frac{1}{2 \pi i} \int_{o_{1}} \frac{d z}{z \bar{z}-1}+\frac{1}{\pi} \int_{\sigma_{1}} \frac{z d \sigma}{(z \bar{z}-1)^{2}}=0
$$

and for the annular area $\sigma_{2}$ bounded by $o_{2}$ and $o_{3}$

$$
\frac{1}{2 \pi i} \int_{o_{3}} \frac{d z}{z \bar{z}-1}-\frac{1}{2 \pi i} \int_{o_{2}} \frac{d z}{z \bar{z}-1}+\frac{1}{\pi} \int_{\sigma_{2}} \frac{z d \sigma}{(z \bar{z}-1)^{2}}=0 .
$$

The sum of these left members will be $R_{\sigma^{\prime}}^{\prime}$, for the area $\sigma^{\prime}$ excluding the poles; this excluded area lies between $o_{1}$ and $o_{2}$.

We wish to determine what happens to $R_{\sigma^{\prime}}^{\prime}$ as $\epsilon \rightarrow 0$ or when $\sigma^{\prime} \rightarrow \sigma$. If we combine the integrals around $o_{1}$ and $o_{2}$ we get zero, since for a fixed $\epsilon$ each integral is zero. In adding the integrals over $\sigma_{1}$ and $\sigma_{2}$ and introducing polar coordinates we find that each integral carries the factor $e^{i \theta} d \theta$ with limits 0 and $2 \pi$. These integrals are evidently zero. From this one must conclude that 


$$
R_{\sigma}^{\prime}=0=\bar{R}_{\sigma}^{\prime}
$$

8. The role of the limit definitions. In reviewing the results obtained under the new definitions it may be noted that every residue over an area was computed through the use of the limit definition. This is evident for the single isolated pole and possibly for $n$ such poles computed as the sum of the residues at each pole. The residue at a point was used directly to determine the residues over an area for the function $f(z)(a z+b \bar{z})^{-1}$. (The difference in the results from those in the Transactions paper were due to the poles outside the circle.) This is also true for the first quadratic function in $\$ 7$. For the discontinuities distributed on the unit circle the limit definition was slightly extended when the circles $o_{1}$ and $o_{2}$ were made to coincide with the unit circle when $\epsilon \rightarrow 0$.

This shows the importance of residues at a point of polygenic functions in the extension to residues over an area. Further, the residues of holomorphic functions become special cases of residues of polygenic functions.

\section{BIBLIOGRAPHY}

1. E. Kasner, General theory of polygenic functions or non-monogenic functions. The derivative congruence of circles, Proc. Nat. Acad. Sci. U.S.A. vol. 14 (1928) pp. 75-82.

2. D. Pompeiu, Sur une classe de fonctions di une variable complex, Rend. Circ. Mat. Palermo vol. 33 (1912) pp. 108-113.

3. V. C. Poor, Residues of polygenic functions, Trans. Amer. Math. Soc. vol. 32 (1930) pp. 216-222.

4. — On the Hamilton differential, Bull. Amer. Math. Soc. vol. 51 (1945) pp. 945-948. 160.

5. - Complex functions possessing differentials, Amer. J. Math. vol. 48 (1946) pp. 147-

UNIVERSITY OF MichigAN, ANn ARbor, Mich. 